\title{
Purines in Pain as a Gliopathy
}

\author{
Giulia Magnit and Stefania Ceruti * \\ Department of Pharmacological and Biomolecular Sciences, Università degli Studi di Milano, Milan, Italy
}

Keywords: microglia, astrocytes, satellite glial cells, A3 adenosine receptors, P2X4 receptors, P2Y12 receptors

\section{INTRODUCTION: WHY IS PAIN CURRENTLY CONSIDERED AS A "GLIOPATHY"}

In the last decades, the hypothesis of the role of purinergic receptors expressed by glial cells in the regulation of pain pathways has become a matter of fact. Although several painful conditions are undoubtedly of neuronal origin, the existence of an altered bidirectional signaling between neurons and activated glial cells has brought to the definition of pain as a "gliopathy" (Ji et al., 2013). In the central nervous system (CNS), neuronal excitability in pain pathways is enhanced by resident microglia, astrocytes, oligodendrocytes, and by infiltrating cells, such as $\mathrm{T}$ cells and macrophages, overall constituting the so-called "neuroimmune interface." Following peripheral nerve damage, spinal cord microglia sense a variety of neuron-derived stimuli leading to their transition toward a reactive state (Grace et al., 2014), whose inhibition through the antibiotic minocycline has proven anti-hyperalgesic and anti-allodynic (Wieseler et al., 2017). Microglia activation is followed by immune cell recruitment and by the appearance of a neurotoxic astrocyte subpopulation, the so-called A1 astrocytes (Liddelow et al., 2017). The extension of microglial and astrocytic processes into and near the synaptic space induces alterations to thousands of synapses, resulting in altered neural networks, maladaptive synaptic reorganization and generating a pro-inflammatory milieu, whose effects can extend for months after the initial nerve damage and guide the transition from acute to chronic pain (Gwak et al., 2017; Skaper et al., 2018). Neuronal firing is also significantly modulated at the sensory ganglion level, where neuronal bodies are surrounded and wrapped by resident satellite glial cells (SCGs), which become activated upon inflammation or nerve damage (Donegan et al., 2013; Magni et al., 2015), release neurotransmitters and chemical mediators and contribute to neuronal sensitization (Spray \& Hanani, 2019). On the other way round, both CNS and PNS sensitized neurons increase their firing and release of neurotransmitters and neuromodulators that act paracrinally on glial cells, giving rise to a vicious and autoamplifying circle. Glial cells become permanently activated as demonstrated both in animal models of chronic pain and in patients (Loggia et al., 2015).

In order to identify new potentially "druggable" targets, research is now focusing on the identification of the whole network of molecules involved in neuron-to-glia communication in pain. In this scenario, multiple adenosine and nucleotide receptors are expressed by glial cells involved in pain transmission (for review see Burnstock, 2017; Magni et al., 2018; Magni \& Ceruti, 2019), and their pharmacological modulation could represent an innovative analgesic strategy. In the next sections we shall contribute with our point of view on the role of P2 nucleotide and P1 adenosine receptors expressed by glial cells in modulating pain transmission, with the aim of identifying new

05 January 202

Accepted: 29 January 2021

Published: 10 March 2021

Citation:

Magni G and Ceruti S (2021) Purines in

Pain as a Gliopathy.

Front. Pharmacol. 12:649807.

doi: 10.3389/fphar.2021.649807 
and more effective approaches to painful conditions with respect to currently available drugs, which mostly target neurons.

\section{PURINERGIC AGENTS TARGETING GLIAL CELLS TO TREAT PAIN: WHAT IS CURRENTLY KNOWN}

\section{Nucleotide Receptors as Key Modulators of Glia Reaction to Injury and Inflammation}

Microglial cells are physiological guardians and modulators of neuronal activity, which can protect brain tissue from excessive neuronal firing. This fundamental function is mostly achieved by the ability of microglia to sense ADP, generated by the rapid breakdown of neuronal ATP released as cotransmitter, through membrane $\mathrm{P}_{2} \mathrm{Y}_{12}$ receptors which in turn control microglia chemotaxis and process dynamics (Badimon et al., 2020) and triggers a cascade of inhibitory events on neurons (Merlini et al., 2021). However, under chronic inflammatory conditions, in the presence of nerve injury or in chronic migraine, microglia become overactivated and shift toward a detrimental state, which involves overexpressed $\mathrm{P} 2 \mathrm{Y}_{12}$ receptors (Jing et al., 2019; Yu et al., 2019). Thus, P2Y 12 selective antagonists (i.e., thienopyridines cangrelor and prasugrel and the more recent nonthienopyridine Ticagrelor), which have been utilized for decades as antiplatelet agents, represent the starting point for the development of a microglia-mediated approach to chronic pain. Actually, a reduction of migraine headache symptoms was observed in patients with patent foramen ovale receiving thienopyridines as antiplatelet therapy (Sommer et al., 2018). These data would need additional confirmations to be exploited in migraine of different origin. Interestingly, $\mathrm{P}_{2} \mathrm{Y}_{12}$ receptors are only expressed by SGCs in sensory ganglia upon nerve injury and their inhibition reduced mechanical and heat hypersensitivity (Sugawara et al., 2017), thus confirming P2 $\mathrm{Y}_{12}$-mediated proalgogenic actions in various painful conditions. Other members of the G protein-coupled P2Y receptor family expressed by SGCs are recruited in chronic pain, including the $\mathrm{P}_{2} \mathrm{Y}_{14}$ (Lin et al., 2019) and the $\mathrm{P}_{2} \mathrm{Y}_{2}$ subtypes (Magni et al., 2015) and likely represent additional interesting targets.

The pivotal role played by ionotropic P2X4 nucleotide receptors in promoting neuronal sensitization after nerve injury has been firmly established over the past 20 years. As mentioned above, nerve injury triggers a complex signaling pathway leading to receptor upregulation in microglia, which in turn promotes the release of BDNF sensitizing second order neurons and causing the development of allodynia, as extensively reviewed elsewhere (Inoue, 2019). It is worth mentioning that the involvement of microglial $\mathrm{P} 2 \mathrm{X} 4$ receptors in pain is sexually dimorphic, occurring in males and not in females (Tam \& Salter, 2020), thus adding further complexity to an already complex scenario and suggesting the issue of sex must be taken into serious consideration in clinical trials.

Upregulation of brainstem microglial P2X4 receptors, which in turn promotes the release of pro-algogenic CGRP, has been also observed in a mouse model of migraine triggered by the recurrent administration of nitroglycerin (Long et al., 2020). Interestingly, novel discoveries have demonstrated a dual neuronal and microglial localization for P2X4 receptors (with controversial data in astrocytes and oligodendrocytes), which are mainly found intracellularly under physiological conditions but whose membrane expression is fostered by pathological stimuli (Duveau et al., 2020). Although the lack of really selective pharmacological tools has sometimes slowed down the discrimination between neuronal and glial P2X4-mediated effects, increased nucleotide concentrations and receptor expression make it possible to foresee a targeted action of P2X4 ligands at sites of pathological neuron-glia crosstalk.

Finally, it has been known for years that nucleotide receptors orchestrate reactive astrogliosis (for review see: Franke \& Illes, 2014), but their role in chronic pain has not been clarified yet.

\section{The Emerging Role for Adenosine in Pain Modulation}

Despite adenosine has been long considered as a simple neuromodulator with the main role to counterbalance and fine-tune nucleotide-mediated actions (Chen et al., 2013), this view has been progressively integrated with the demonstration that adenosine receptors, widely expressed throughout the body, play fundamental roles in many physiological and pathological conditions (Borea et al., 2018). This is particularly true for pathologies in which the local extracellular concentrations of nucleotides (and of their breakdown products nucleosides) rise several folds, such as tissue damage and hypoxia, but also during increased neuronal firing as in epilepsy and chronic pain. Many clinical trials involving adenosine receptor ligands have been carried out so far in various pathological conditions, but only a few compounds have been approved since 1990, i.e., the $\mathrm{A}_{2 \mathrm{~A}}$ receptor agonist Regadenoson as pharmacological stress agent for myocardial perfusion imaging (MPI) and the selective $A_{2 A}$ receptor antagonist Istradefylline as an add-on to levodopa/ carbidopa treatment for Parkinson's disease (Jacobson et al., 2019; Chen \& Cunha, 2020).

Concerning pain, adenosine receptors are expressed by glial cells and participate to their communication with neurons both in the CNS and in peripheral ganglia (Agostinho et al., 2020). Therefore, these receptors undoubtedly represent a promising approach to manage pain conditions characterized by a dysregulated cross-talk among different cell types. Available and sometimes contradictory data on the antinociceptive effects exerted by the administration of adenosine ligands in several preclinical models of acute and chronic pain have been recently extensively reviewed (Vincenzi et al., 2020). We shall now briefly highlight the most promising hints for their possible exploitation in humans as well.

The very first hypothesis of a contribution of adenosine receptors in pain was based on the role of the $A_{1}$ and $A_{2 A}$ subtypes but, despite their efficacy in preclinical studies, selective agonists could not be included in clinical trials due to significant cardiovascular side effects (Jacobson et al., 2020). Nowadays, the most promising compounds under 


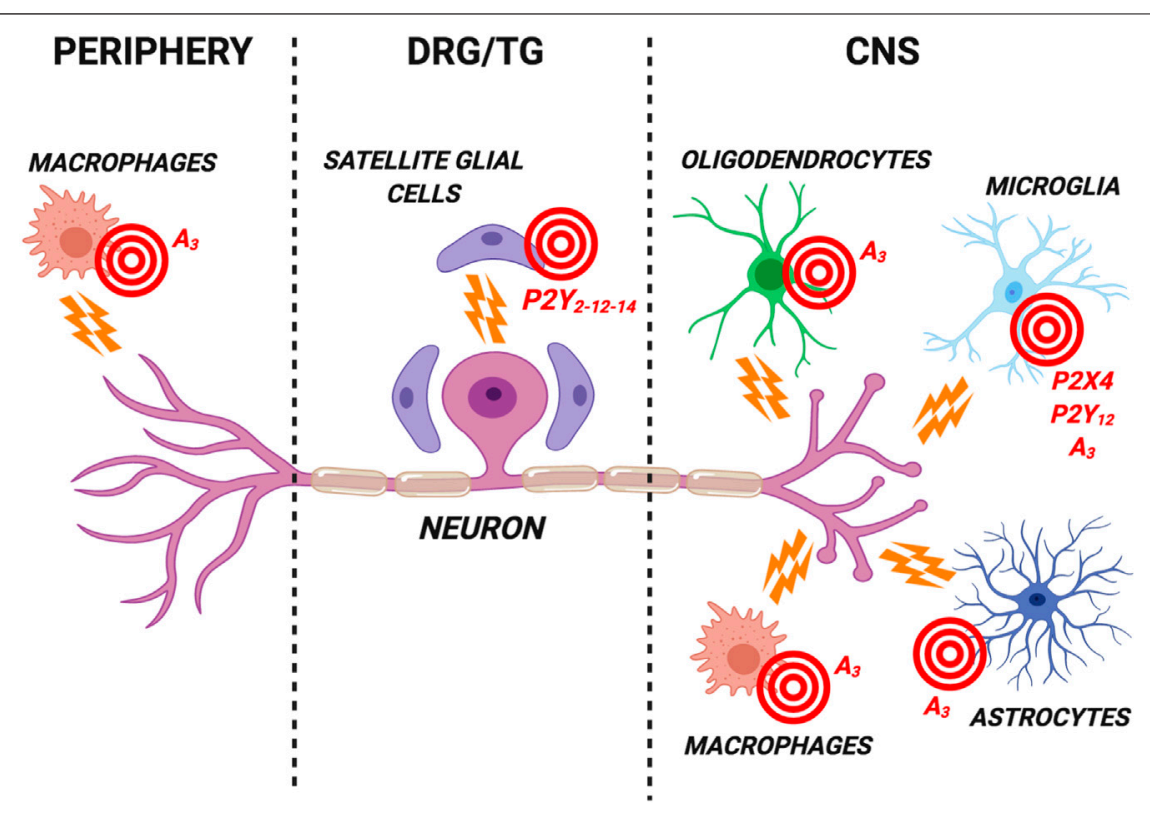

FIGURE 1 | Schematic representation of the most promising purinergic targets for the development of new analgesics expressed by glial cells in the central nervous system (CNS), in dorsal root ganglia/trigeminal ganglia (DRG/TG) and by peripheral cells. These nucleotide (i.e., the P2 $\mathrm{Y}_{12}$ and P2X4 subtypes) and adenosine (i.e., the $\mathrm{A}_{3}$ subtype) receptors are involved in the modulation of neuronal firing in chronic pain conditions. Receptor antagonists are likely to represent the best analgesic option targeting P2Y ${ }_{12}$ and $\mathrm{P} 2 \mathrm{X} 4$ subtypes. Conversely, selective $\mathrm{A}_{3}$ receptor agonists have proved effective in reducing pain in several preclinical models. See text for details. Created with BioRender.com.

development are $\mathrm{A}_{3}$ receptor agonists: preclinical data showed that they are able to revert signaling dysregulation in cancer, autoimmune disorders and different pain conditions (Doyle et al., 2020). When compared to $A_{1}$ and $A_{2 A}$ receptors, $A_{3}$ receptors have low CNS expression levels, but are highly expressed in human immune and glial cells, including astrocytes, oligodendrocytes, microglia/macrophages and endothelial cells (Zhang et al., 2016), i.e., cell populations that are mostly involved in chronic painful conditions. In the last five years, the $\mathrm{A}_{3}$ receptor subtype has progressively emerged as innovative target for the control of pain, also thanks to the availability of selective pharmacological modulators, starting from IB-MECA and Cl-IB-MECA up to new more selective and potent agonists, including MRS5698 and MRS5980, which proved their efficacy in preclinical models of pain, including formalin-induced inflammatory pain and diabetic neuropathy (Magni et al., 2018; Jacobson et al., 2019; 2020).

Noteworthy, it has been demonstrated that dysregulation of $\mathrm{A}_{3}$ adenosine receptors recruitment is at the basis of chemotherapy-induced pain (Wahlman et al., 2018), but $\mathrm{A}_{3}$ receptor agonists do not interfere with antitumor effects of widely used chemotherapeutics; rather, they act as antitumor agents themselves. In fact, Cl-IB-MECA is currently in Phase II clinical trials for hepatocellular carcinoma as anticancer agent, and evidence suggest that activation of $\mathrm{A}_{3}$ receptors could provide dual benefits in the treatment of a variety of cancerrelated pain states (Jacobson et al., 2020). As for chronic pain states, $\mathrm{A}_{3}$ agonists can be used as a monotherapy or in combination with opioids to improve their safety and efficacy against chronic pain, without reducing opioid's antinociceptive effects (Doyle et al., 2020). Taken together, available data strongly support the further development of selective $\mathrm{A}_{3}$ receptor agonists for the treatment of different types of pain syndromes.

\section{CONCLUSION}

As for most of the discoveries on the purinergic system, the hypothesis of a purinergic modulation of pain plunges its roots in the intuitions of Geoffrey Burnstock who highlighted the P2X3 neuronal subtype as one of the fundamental receptors controlling visceral, inflammatory and neuropathic pain (Jarvis, 2020). However, several P2X3-selective antagonists have failed in clinical trials possibly due to limited bioavailability and difficulties in reaching effective concentrations at the site of action. Gefapixant, one recently developed compound which has been named after Geoff who contributed to its development, is currently undergoing clinical trials for chronic cough (Jarvis, 2020), thus confirming the key contribution of nucleotide receptors to sensory transmission. The main difficulty in translating exciting preclinical data summarized above on the purinergic modulation of glial cells to effective drugs (Figure 1) resides in the high complexity of this signaling system, on its intrinsic variability among different species, on its huge numbers of ligands, enzymes, transporters and receptor subtypes with a widespread expression which play key roles in many 
physiopathological conditions, thus leading to possible side effects. One recent example is represented by the demonstration that $A_{1}$ receptors expressed by SCGs in rat DRGs reversed inflammation-induced mechanical allodynia (Xie et al., 2020). The use of agents targeting this receptor subtype would necessarily face the appearance of significant cardiovascular side effects, as long as locally-acting ligands or drug delivery strategies are applied. The use of allosteric modulators and/or partial agonists would be an additional strategy to selectively target receptors at affected sites.

Overall, the development of the first "purinergic analgesic" could be accelerated by already ongoing clinical studies for different indications. For example, selective and potent $\mathrm{A}_{3}$ adenosine receptor agonists are currently undergoing clinical trials for psoriasis (Phase III; Piclidenoson, CanFite BioPharma) and liver diseases (Phase II; Namodenoson, CanFite BioPharma). A phase I clinical trial with the P2X4 antagonist NC-2600 has been recently concluded by Nippon Chemiphar, showing safety and no serious side effects. Finally, novel pharmacological entities have now started to emerge which could overcome the evident difficulties in the successful delivery of "classical" chemical ligands. Monoclonal antibodies acting on human and mouse P2X4 have been synthesized showing

\section{REFERENCES}

Agostinho, P., Madeira, D., Dias, L., Simões, A. P., Cunha, R. A., and Canas, P. M. (2020). Purinergic signaling orchestrating neuron-glia communication. Pharmacol. Res. 162, 105253. doi:10.1016/j.phrs.2020.105253

Badimon, A., Strasburger, H. J., Ayata, P., Chen, X., Nair, A., Ikegami, A., et al. (2020). Negative feedback control of neuronal activity by microglia. Nature 586 (7829), 417-423. doi:10.1038/s41586-020-2777-8

Borea, P. A., Gessi, S., Merighi, S., Vincenzi, F., and Varani, K. (2018). Pharmacology of adenosine receptors: the state of the art. Physiol. Rev. 98 (3), 1591-1625. doi:10.1152/physrev.00049.2017

Burnstock, G. (2017). Purinergic signalling: therapeutic developments. Front. Pharmacol. 8, 661. doi:10.3389/fphar.2017.00661

Chen, J. F., and Cunha, R. (2020). The belated US FDA approval of the adenosine A2A receptor antagonist istradefylline for treatment of Parkinson's disease. Purinergic Signal. 16 (2), 167-174. doi:10.1007/ s11302-020-09694-2

Chen, J. F., Eltzschig, H. K., and Fredholm, B. B. (2013). Adenosine receptors as drug targets--what are the challenges?. Nat. Rev. Drug Discov. 12 (4), 265-286. doi:10.1038/nrd3955

Donegan, M., Kernisant, M., Cua, C., Jasmin, L., and Ohara, P. T. (2013). Satellite glial cell proliferation in the trigeminal ganglia after chronic constriction injury of the infraorbital nerve. Glia 61, 2000-2008. doi:10. 1002/glia.22571

Doyle, T. M., Largent-Milnes, T. M., Chen, Z., Staikopoulos, V., Esposito, E., Dalgarno, R., et al. (2020). Chronic morphine-induced changes in signaling at the A3 adenosine receptor contribute to morphine-induced hyperalgesia, tolerance, and withdrawal. J. Pharmacol. Exp. Ther. 374 (2), 331-341. doi:10.1124/jpet.120.000004

Duveau, A., Bertin, E., and Boué-Grabot, E. (2020). Implication of neuronal versus microglial P2X4 receptors in central nervous system disorders. Neurosci. Bull. 36 (11), 1327-1343. doi:10.1007/s12264-020-00570-y

Franke, H., and Illes, P. (2014). Nucleotide signaling in astrogliosis. Neurosci. Lett. 565, 14-22. doi:10.1016/j.neulet.2013.09.056

Grace, P. M., Hutchinson, M. R., Maier, S. F., and Watkins, L. R. (2014). Pathological pain and the neuroimmune interface. Nat. Rev. Immunol. 14, 217-231. doi:10.1038/nri3621 enhanced blood-brain barrier permeability and which can be systemically delivered (Williams et al., 2019). The observed longlasting analgesic effect in mouse models of neuropathic pain gives hope for their future clinical exploitation.

\section{AUTHOR CONTRIBUTIONS}

GM (ORCID id: 0000-0003-0995-8947) drafted the Introduction and adenosine sections, and prepared Figure 1. SC drafted nucleotide and Conclusions sections and revised the whole manuscript. Both authors approved the final version of the paper.

\section{FUNDING}

Authors' work has been supported by the Fondazione Italiana Sclerosi Multipla (FISM; grant \#2016/R/7 to SC). Our department has been awarded with the "Department of Excellence" grant (2018-2022) by the Italian Ministry of University and Research (MIUR). Authors acknowledge support from the Università degli Studi di Milano through the APC initiative.

Gwak, Y. S., Hulsebosch, C. E., and Leem, J. W. (2017). Neuronal-glial interactions maintain chronic neuropathic pain after spinal cord injury. Neural Plast. 2017, 2480689. doi: $10.1155 / 2017 / 2480689$

Inoue, K. (2019). Role of the P2X4 receptor in neuropathic pain. Curr. Opin. Pharmacol. 47, 33-39. doi:10.1016/j.coph.2019.02.001

Jacobson, K. A., Giancotti, L. A., Lauro, F., Mufti, F., and Salvemini, D. (2020). Treatment of chronic neuropathic pain: purine receptor modulation. Pain 161 (7), 1425-1441. doi:10.1097/j.pain.0000000000001857

Jacobson, K. A., Tosh, D. K., Jain, S., and Gao, Z. G. (2019). Historical and current adenosine receptor agonists in preclinical and clinical development. Front. Cell. Neurosci. 13, 124. doi:10.3389/fncel.2019.00124

Jarvis, M. F. (2020). Geoffery Burnstock's influence on the evolution of P2X3 receptor pharmacology. Purinergic Signal. [Epub ahead of print]. doi:10.1007/ s11302-020-09744-9Online

Ji, R. R., Berta, T., and Nedergaard, M. (2013). Glia and pain: is chronic pain a gliopathy?. Pain 154 (Suppl. 1), S10-S28. doi:10.1016/j.pain.2013.06.022

Jing, F., Zhang, Y., Long, T., He, W., Qin, G., Zhang, D., et al. (2019). P2Y12 receptor mediates microglial activation via RhoA/ROCK pathway in the trigeminal nucleus caudalis in a mouse model of chronic migraine. J. Neuroinflammation 16 (1), 217. doi:10.1186/s12974-019-1603-4

Liddelow, S. A., Guttenplan, K. A., Clarke, L. E., Bennett, F. C., Bohlen, C. J., Schirmer, L., et al. (2017). Neurotoxic reactive astrocytes are induced by activated microglia. Nature 541 (7638), 481-487. doi:10.1038/ nature 21029

Lin, J., Zhang, Y. Y., Liu, F., Fang, X. Y., Liu, M. K., Huang, C. L., et al. (2019). The $\mathrm{P} 2 \mathrm{Y} 14$ receptor in the trigeminal ganglion contributes to the maintenance of inflammatory pain. Neurochem. Int. 131, 104567. doi:10.1016/j.neuint.2019. 104567

Loggia, M. L., Chonde, D. B., Akeju, O., Arabasz, G., Catana, C., Edwards, R. R., et al. (2015). Evidence for brain glial activation in chronic pain patients. Brain 138 (Pt 3), 604-615. doi:10.1093/brain/awu377

Long, T., He, W., Pan, Q., Zhang, S., Zhang, D., Qin, G., et al. (2020). Microglia $\mathrm{P} 2 \mathrm{X} 4 \mathrm{R}-\mathrm{BDNF}$ signalling contributes to central sensitization in a recurrent nitroglycerin-induced chronic migraine model. J. Headache Pain 21 (1), 4. doi:10.1186/s10194-019-1070-4

Magni, G., and Ceruti, S. (2019). The role of adenosine and P2Y receptors expressed by multiple cell types in pain transmission. Brain Res. Bull. 151, 132-143. doi:10.1016/j.brainresbull.2019.02.011 
Magni, G., Merli, D., Verderio, C., Abbracchio, M. P., and Ceruti, S. (2015). P2Y2 receptor antagonists as anti-allodynic agents in acute and sub-chronic trigeminal sensitization: role of satellite glial cells. Glia 63 (7), 1256-1269. doi:10.1002/glia.22819

Magni, G., Riccio, D., and Ceruti, S. (2018). Tackling chronic pain and inflammation through the purinergic system. Curr. Med. Chem. 25 (32), 3830-3865. doi:10.2174/0929867324666170710110630

Merlini, M., Rafalski, V. A., Ma, K., Kim, K. Y., Bushong, E. A., Rios Coronado, P. E., et al. (2021). Microglial gi-dependent dynamics regulate brain network hyperexcitability. Nat. Neurosci. 24 (1), 19-23. doi:10.1038/s41593-020-00756-7

Skaper, S. D., Facci, L., Zusso, M., and Giusti, P. (2018). An inflammation-centric view of neurological disease: beyond the neuron. Front. Cell. Neurosci. 12, 72. doi:10.3389/fncel.2018.00072

Sommer, R. J., Nazif, T., Privitera, L., and Robbins, B. T. (2018). Retrospective review of thienopyridine therapy in migraineurs with patent foramen ovale. Neurology 91, 1002-1009. doi:10.1212/WNL.0000000000006572

Spray, D. C., and Hanani, M. (2019). Gap junctions, pannexins and pain. Neurosci. Lett. 695, 46-52. doi:10.1016/j.neulet.2017.06.035

Sugawara, S., Okada, S., Katagiri, A., Saito, H., Suzuki, T., Komiya, H., et al. (2017). Interaction between calcitonin gene-related peptide-immunoreactive neurons and satellite cells via P2Y12 R in the trigeminal ganglion is involved in neuropathic tongue pain in rats. Eur. J. Oral Sci. 125 (6), 444-452. doi:10.1111/eos.12382

Tam, T. H., and Salter, M,W. (2020). Purinergic signalling in spinal pain processing. Purinergic Signal. [Epub ahead of print]. doi:10.1007/s11302-020-09748-5Online

Vincenzi, F., Pasquini, S., Borea, P. A., and Varani, K. (2020). Targeting adenosine receptors: a potential pharmacological avenue for acute and chronic pain. Int. J. Mol. Sci. 21 (22), 8710. doi:10.3390/ijms21228710

Wahlman, C., Doyle, T. M., Little, J. W., Luongo, L., Janes, K., Chen, Z., et al. (2018). Chemotherapy-induced pain is promoted by enhanced spinal adenosine kinase levels through astrocyte-dependent mechanisms. Pain 159 (6), 1025-1034. doi:10.1097/j.pain.0000000000001177
Wieseler, J., Ellis, A., McFadden, A., Stone, K., Brown, K., Cady, S., et al. (2017) Supradural inflammatory soup in awake and freely moving rats induces facial allodynia that is blocked by putative immune modulators. Brain Res. 1664, 87-94. doi:10.1016/j.brainres.2017.03.011

Williams, W. A., Linley, J. E., Jones, C. A., Shibata, Y., Snijder, A., Button, J., et al. (2019). Antibodies binding the head domain of P2X4 inhibit channel function and reverse neuropathic pain. Pain 160 (9), 1989-2003. doi:10.1097/j.pain. 0000000000001587

Xie, A. X., Madayag, A., Minton, S. K., McCarthy, K. D., and Malykhina, A. P. (2020). Sensory satellite glial Gq-GPCR activation alleviates inflammatory pain via peripheral adenosine 1 receptor activation. Sci. Rep. 10 (1), 14181. doi:10. 1038/s41598-020-71073-Z

Yu, T., Zhang, X., Shi, H., Tian, J., Sun, L., Hu, X., et al. (2019). P2Y12 regulates microglia activation and excitatory synaptic transmission in spinal lamina II neurons during neuropathic pain in rodents. Cell Death Dis. 10, 165. doi:10. 1038/s41419-019-1425-4

Zhang, Y., Sloan, S. A., Clarke, L. E., Caneda, C., Plaza, C. A., Blumenthal, P. D., et al. (2016). Purification and characterization of progenitor and mature human astrocytes reveals transcriptional and functional differences with mouse. Neuron 89, 37-53. doi:10.1016/j.neuron.2015.11.013

Conflict of Interest: The authors declare that the research was conducted in the absence of any commercial or financial relationships that could be construed as a potential conflict of interest.

Copyright (c) 2021 Magni and Ceruti. This is an open-access article distributed under the terms of the Creative Commons Attribution License (CC BY). The use, distribution or reproduction in other forums is permitted, provided the original author(s) and the copyright owner(s) are credited and that the original publication in this journal is cited, in accordance with accepted academic practice. No use, distribution or reproduction is permitted which does not comply with these terms. 